\title{
Structural Basis for Substrate Promiscuity of dCK
}

\author{
Elisabetta Sabini ${ }^{1}$, Saugata Hazra ${ }^{1}$, Stephan Ort $^{2}$, Manfred Konrad ${ }^{2}$ \\ and Arnon Lavie ${ }^{1 *}$
}

\author{
${ }^{1}$ Department of Biochemistry \\ and Molecular Genetics, \\ University of Illinois at Chicago, \\ 900 South Ashland Avenue, \\ Chicago, IL 60607, USA \\ ${ }^{2}$ Max-Planck-Institute for \\ Biophysical Chemistry, \\ D-37070, Göttingen, Germany
}

Received 27 November 2007; received in revised form 12 February 2008; accepted 14 February 2008 Available online 3 March 2008

\begin{abstract}
Deoxycytidine kinase $(\mathrm{dCK})$ is an essential nucleoside kinase critical for the production of nucleotide precursors for DNA synthesis. This enzyme catalyzes the initial conversion of the nucleosides deoxyadenosine (dA), deoxyguanosine $(\mathrm{dG})$, and deoxycytidine $(\mathrm{dC})$ into their monophosphate forms, with subsequent phosphorylation to the triphosphate forms performed by additional enzymes. Several nucleoside analog prodrugs are dependent on $\mathrm{dCK}$ for their pharmacological activation, and even nucleosides of the non-physiological L-chirality are phosphorylated by $\mathrm{dCK}$. In addition to accepting $\mathrm{dC}$ and purine nucleosides (and their analogs) as phosphoryl acceptors, dCK can utilize either ATP or UTP as phosphoryl donors. To unravel the structural basis for substrate promiscuity of $\mathrm{dCK}$ at both the nucleoside acceptor and nucleotide donor sites, we solved the crystal structures of the enzyme as ternary complexes with the two enantiomeric forms of $\mathrm{dA}$ (D-dA, or L-dA), with either UDP or ADP bound to the donor site. The complexes with UDP revealed an open state of $\mathrm{dCK}$ in which the nucleoside, either D-dA or L-dA, is surprisingly bound in a manner not consistent with catalysis. In contrast, the complexes with ADP, with either D-dA or L-dA, adopted a closed and catalytically competent conformation. The differential states adopted by $\mathrm{dCK}$ in response to the nature of the nucleotide were also detected by tryptophan fluorescence experiments. Thus, we are in the unique position to observe differential effects at the acceptor site due to the nature of the nucleotide at the donor site, allowing us to rationalize the different kinetic properties observed with UTP to those with ATP.
\end{abstract}

(C) 2008 Elsevier Ltd. All rights reserved.

Keywords: deoxycytidine kinase; phosphoryl transfer; L-nucleosides; deoxyadenosine; crystal structure gemcitabine, cladribine and fludarabine) and viral infections (e.g., lamivudine and emtracitabine). Interestingly, both ATP and UTP can act as phosphoryl donors, and it has been suggested that UTP is the in vivo relevant donor. ${ }^{1}$ In addition, $\mathrm{dCK}$ has the ability to phosphorylate NAs of the non-physiological L-chirality.

The low substrate selectivity of dCK at both the donor and acceptor sites, allowing molecules that differ in size, shape, chirality, and hydrogen bonding pattern to be substrates, suggests that the enzyme can adjust its conformation to preserve its catalytic power. However, before this work our understanding of how dCK maintains its enzymatic activity with such diverse substrates has been limited. For example, previous structures of $\mathrm{dCK}$ contained pyrimidine nucleosides at the acceptor site, ${ }^{3-5}$ except for the sole structure of
${ }^{*}$ Corresponding author. E-mail address: Lavie@uic.edu.

Abbreviations used: dCK, deoxycytidine kinase; NA, nucleoside analog; $\mathrm{C}_{4} \mathrm{~S}$, a human $\mathrm{dCK}$ variant in which four cysteine residues (C9, C45, C59, and C146) were exchanged with serine; WT, wild type. 
$\mathrm{dCK}$ in complex with the purine clofarabine, a structure solved at medium resolution and that exhibits very high $B$-factors. ${ }^{6}$ Additionally, the only structure of dCK with a uridine nucleotide at the donor site was solved at low resolution and employed a dCK variant lacking its insert region. ${ }^{7}$ Lastly, the recently reported structural analyses of phosphorylation of L-nucleosides by dCK were limited to pyrimidine nucleosides. ${ }^{4,5}$

We set out to address the following questions: (1) what structural adjustments take place upon binding of the physiological purine substrate $\mathrm{dA}$, in comparison to the pyrimidine $\mathrm{dC}$ ? (2) How is the nature of the nucleotide at the donor site sensed by the enzyme? (3) How do the acceptor and donor binding sites communicate, as suggested by the fact that the donor identity affects the kinetic parameters of the nucleoside, and vice versa? (4) What are the structural ramifications of binding to the non-physiological Lform of $\mathrm{dA}$ ? To address these questions, we solved the three-dimensional structures of dCK in complex with the purine nucleoside $\mathrm{dA}$ (in the form of either D$\mathrm{dA}$ or L-dA) at the nucleoside-binding site, and either ADP or UDP at the phosphate donor site. Apart from supplying the first view of $\mathrm{dCK}$ bound to one of its physiological purine substrates, a surprising result of this work was the detection of an open dCK conformation in the presence of uridine nucleotides. This state, corroborated by tryptophan fluorescence studies, rationalizes the different kinetic behavior observed for dCK when UTP is exchanged for ATP. This work increases our understanding of the structural basis for the substrate promiscuity of dCK.

\section{Results and Discussion}

Structural work on human dCK has been severely hampered by the difficulty of obtaining diffractionquality crystals. For example, whereas wild type (WT) dCK forms good crystals as a ternary complex with ADP at the donor site and various pyrimidine nucleosides at the acceptor site, we could not obtain crystals when UDP was used instead of ADP. This was an early indication that dCK adjusts its conformation according to the nature of the nucleotides present. A major effort was initiated to identify a variant of the enzyme that would form good crystals with a wider set of substrates. We speculated that one possible hindrance to crystallization was the presence of surface cysteine residues. Therefore, a variant of $\mathrm{dCK}$ that has four of its six cysteine residues mutated to serine was generated. Extensive structural and kinetic analysis of this socalled $\mathrm{C}_{4} \mathrm{~S}-\mathrm{dCK}$ variant revealed that it is a good analog of WT-dCK. ${ }^{4}$ For example, several structures previously solved with WT-dCK were repeated with the $\mathrm{C}_{4} \mathrm{~S}$ variant. Comparison of these structures revealed an identical overall fold, and an identical nucleoside and nucleotide conformation. ${ }^{4,5}$ Importantly, unlike WT-dCK, the $\mathrm{C}_{4} \mathrm{~S}-\mathrm{dCK}$ variant exhibits an improved propensity for crystallization. Three of the structures of $\mathrm{dCK}$ reported here employed the
$\mathrm{C}_{4} \mathrm{~S}$-variant. One, the D-dA/ADP complex, crystallized when an additional surface residue, Glu247, was mutated to alanine (see Materials and Methods). Kinetic and fluorescence studies were done on both the $\mathrm{C}_{4} \mathrm{~S}$ and the WT-dCK proteins.

Here, we present four ternary complexes of dCK that contain either the $\mathrm{D}$ - or the L-form of $\mathrm{dA}$ at the nucleoside acceptor site, plus either ADP or UDP at the phosphoryl donor site. For clarity, we specifically designate the chirality of the nucleosides discussed (e.g. D-dA or L-dA). Data collection and refinement statistics for the D-dA/ADP (to $2.3 \AA$ resolution), L-dA/ADP (2.2 ̊̊), D-dA/UDP (1.77 ̊̊), and L-dA/UDP (1.77 $\AA$ ) complexes are presented in Table 1.

\section{Side chain adjustments of active site residues allows dCK to bind either purine or pyrimidine nucleosides: comparison of the D-dA/ADP to the D-dC/ADP complexes}

One of the goals of this work was to elucidate the structural properties of dCK that allow this nucleoside kinase to phosphorylate both purine and pyrimidine substrates. Comparison of ternary D$\mathrm{dA} / \mathrm{ADP}$ (i.e. a purine at the acceptor site) and D$\mathrm{dC} / \mathrm{ADP}$ (i.e. a pyrimidine at the acceptor site; PDB ID 1P60) complex structures shows that the overall fold of the enzyme is very similar (Fig. 1a). There are no significant differences in the positioning of mainchain atoms (rmsd between the D-dA/ADP structure and the D-dC/ADP structure is $0.29 \AA$ on $239 \mathrm{C}_{\alpha}$ atoms). An additional correspondence lies in the insert region. In the previous structure of $\mathrm{dCK}$ in complex with D-dC/ADP, we could model the insert region in only one of the two molecules that build the asymmetric unit. ${ }^{3}$ In the D-dA/ADP complex, which also contains a dimer in the asymmetric unit, we observe excellent electron density for the insert region for one of the monomers, and in a conformation identical with that in the D-dC/ADP complex.

While no gross conformational difference exists between the pyrimidine (D-dC) and the purine (D$\mathrm{dA}$ ) bound states, several active site residues do slightly adjust their position according to the shape, size, and hydrogen bonding capabilities of the substrate. For example, Asp133 is closer to the amino group of the adenine moiety compared to the amino group of the cytosine ring $(2.5 \AA$ versus $2.9 \AA$ A) and concomitantly Arg104 readjusts its position to make a tighter interaction with Asp133 (Fig. 1b). Also, in the D-dA structure, the side chain of Gln97 rotates slightly, keeping the oxygen of the amide group in the same position while adjusting the position of the NH2-group slightly in order to accommodate atom N1 of dA (see Supplementary Data Fig. S1). The larger purine ring in D-dA results in the displacement of a water molecule that otherwise bridges the carbonyl group of $\mathrm{D}-\mathrm{dC}$ and Gln97. On the other hand, the water molecule bridging $\mathrm{D}-\mathrm{dC}$ and Tyr86 is retained in the D-dA structure. For this, D-dA uses its ring N3 instead of the carbonyl group used by $\mathrm{D}-\mathrm{dC}$. This water- 
Table 1. Data collection and refinement statistics

\begin{tabular}{|c|c|c|c|c|}
\hline dCK mutant & $\mathrm{C}_{4} \mathrm{~S}+\mathrm{E} 247 \mathrm{~A}$ & $\mathrm{C}_{4} \mathrm{~S}$ & $\mathrm{C}_{4} \mathrm{~S}$ & $\mathrm{C}_{4} \mathrm{~S}$ \\
\hline Complex & $\mathrm{D}-\mathrm{dA} / \mathrm{ADP}$ & L-dA/ADP & D-dA/UDP & L-dA/UDP \\
\hline PDB codes & 2ZII3 & 2ZI4 & 2ZI6 & 2ZI5 \\
\hline $\begin{array}{l}\text { Crystal growth } \\
\text { temperauture }\left({ }^{\circ} \mathrm{C}\right)\end{array}$ & 22 & 22 & 12 & 12 \\
\hline \multirow[t]{2}{*}{ X-ray source and detector } & $\begin{array}{c}\text { APS } \\
\text { (SERCAT ID-22) }\end{array}$ & $\begin{array}{l}\text { Rotating anode } \\
\text { RAXIS-IV }\end{array}$ & $\begin{array}{l}\text { Rotating anode } \\
\text { RAXIS-IV }\end{array}$ & $\begin{array}{l}\text { Rotating anode } \\
\text { RAXIS-IV }\end{array}$ \\
\hline & MARCCD 300 & & & \\
\hline Wavelength (Å) & 1.0 & 1.5418 & 1.5418 & 1.5418 \\
\hline Temperature (K) & 100 & 100 & 100 & 100 \\
\hline Resolution ${ }^{\mathrm{a}}(\AA ̊)$ & $2.3(2.3-2.4)$ & $1.76(1.76-1.87)$ & $1.77(1.77-1.87)$ & $1.77(1.77-1.87)$ \\
\hline \multicolumn{5}{|l|}{ No. reflections } \\
\hline Observed & 112,092 & 274,380 & 528,419 & 76,8651 \\
\hline Unique & 23,174 & 30,274 & 109,802 & 110,589 \\
\hline Completeness (\%) & $93.0(81.8)$ & 99.5 (97.6) & $98.6(93.0)$ & $99.0(95.4)$ \\
\hline$R_{\mathrm{sym}}(\%)$ & $13.2(54.5)$ & $11.1(34.6)$ & $7.1(41.4)$ & $6.7(52.2)$ \\
\hline$I / \sigma(I)$ & $8.4(2.6)$ & $13.9(6.0)$ & $13.4(3.0)$ & $16.6(3.1)$ \\
\hline Space group & $\mathrm{C} 222_{1}$ & $P 4_{3} 2_{1} 2$ & $\mathrm{C} 222_{1}$ & $C 222_{1}$ \\
\hline \multicolumn{5}{|l|}{ Unit cell } \\
\hline$a(\AA)$ & 52.44 & 79.73 & 138.25 & 138.36 \\
\hline$b(\AA)$ & 133.29 & 79.73 & 138.51 & 138.65 \\
\hline$c(\AA)$ & 156.73 & 93.53 & 118.67 & 119.01 \\
\hline Refinement program & REFMAC5 & REFMAC5 & SHELXL & SHELXL \\
\hline Twinning fraction & - & - & 0.48265 & 0.50115 \\
\hline \multicolumn{5}{|l|}{ Refinement statistics } \\
\hline$R_{\text {cryst }}(\%)$ & 20.8 & 17.9 & 20.8 & 21.9 \\
\hline$R_{\text {free }}(\%)$ & 30.6 & 25.7 & 28.3 & 29.3 \\
\hline Resolution range $(\AA)$ & $30-2.3$ & $30-2.2$ & $30-1.77$ & $30-1.77$ \\
\hline Molecules per a.u. & 2 & 1 & 4 & 4 \\
\hline \multicolumn{5}{|l|}{ Number of atoms } \\
\hline Protein & 1996,1860 & 1880 & $\begin{array}{l}1807,1867 \\
1808,1797\end{array}$ & $\begin{array}{l}1837,1856, \\
1827,1813\end{array}$ \\
\hline D-dA/L-dA & $18 \times 2$ & $18 \times 1$ & $18 \times 4$ & $18 \times 4$ \\
\hline ADP/UDP & $27 \times 2$ & $27 \times 1$ & $25 \times 4$ & $25 \times 4$ \\
\hline Water & 104 & 86 & 165 & 211 \\
\hline \multicolumn{5}{|l|}{ r.m.s. deviation from ideal } \\
\hline Bond lengths $(\AA)$ & 0.015 & 0.014 & 0.007 & 0.006 \\
\hline Bond angles (deg) & 1.738 & 1.572 & - & - \\
\hline Angle distance $(\mathrm{A})$ & - & - & 0.027 & 0.023 \\
\hline \multicolumn{5}{|l|}{ Average $B$-factors $\left(\AA^{2}\right) /$ chain } \\
\hline Protein & 39,41 & 33 & $29,27,30,27$ & $35,32,35,33$ \\
\hline D-dA/L-dA & 32,33 & 20 & $23,18,30,20$ & $33,35,35,34$ \\
\hline $\mathrm{ADP} / \mathrm{UDP}$ & 27,29 & 22 & 24,$27 ; 26 ; 23$ & $31,28,36,32$ \\
\hline Water molecules & 39 & 36 & 27 & 34 \\
\hline \multicolumn{5}{|l|}{ Ramachandran plot regions } \\
\hline Most favored (\%) & 91.5 & 93.2 & 87.2 & 86.1 \\
\hline Additionally allowed (\%) & 7.3 & 6.3 & 11.7 & 12.9 \\
\hline Generously allowed (\%) & 0.9 & 0.5 & 0.9 & 0.9 \\
\hline Disallowed (\%) & 0.2 & 0.0 & 0.3 & 0.1 \\
\hline
\end{tabular}

mediated nucleoside-protein interaction is important for positioning of the side chain of Tyr86. The position of this residue, which also interacts with the $3^{\prime}-\mathrm{OH}$ group of the nucleoside, seems to be critical for the ability of $\mathrm{dCK}$ to adopt a closed and active conformation (see below).

Interestingly, when it comes to the binding and orientation of the nucleosides, the amino groups of the bases (N4 in dC, N6 in dA) are in nearly identical positions (arrow, Fig. 1b), and so are the respective $3^{\prime}$ - and the $5^{\prime}-\mathrm{OH}$ groups of the sugar moieties. This allows the protein to maintain a similar hydrogen bonding pattern irrespective to the base nature of the nucleoside, and to correctly orient and activate the 5 '-OH group for nucleophilic attack on the phosphoryl donor. In summary, in order to accommodate the larger purine substrates, $\mathrm{dCK}$ adjusts the position of active site residues so that homologous interactions as seen with pyrimidines substrates can be made.

\section{The nature of the nucleotide at the phosphoryl donor site determines the conformation of dCK: comparison of the D-dA/ADP to D-dA/UDP structures}

In contrast to a preserved enzyme conformation between the $\mathrm{D}-\mathrm{dC}$ and the $\mathrm{D}-\mathrm{dA}$ complexes, both with ADP at the phosphoryl donor site, comparison of the D-dA/ADP to the D-dA/UDP structures (i.e., the same purine at the nucleoside site, but a different diphosphate at the donor site) shows that binding of different nucleotides results in differences in the overall-fold of the protein, leading to an rmsd value 
(a)

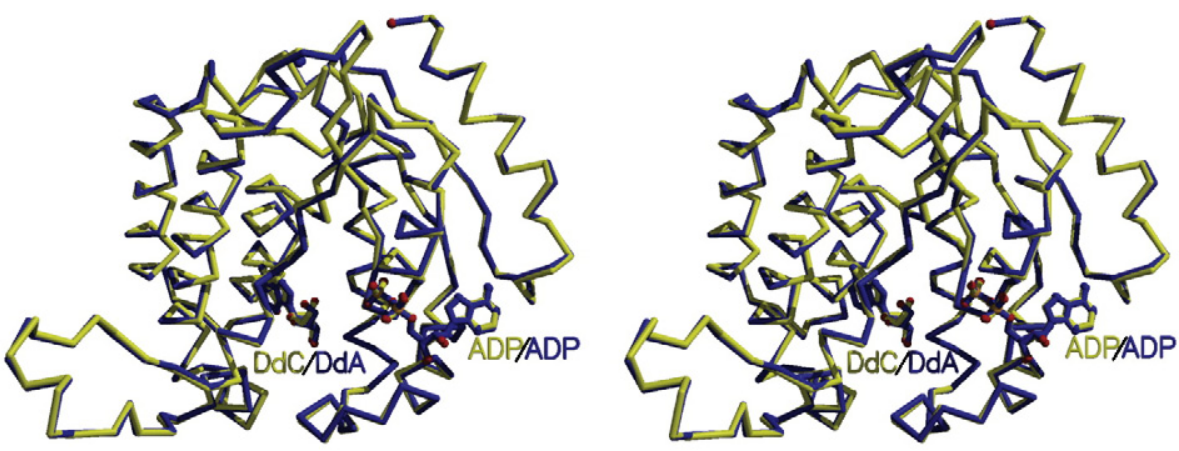

(b)

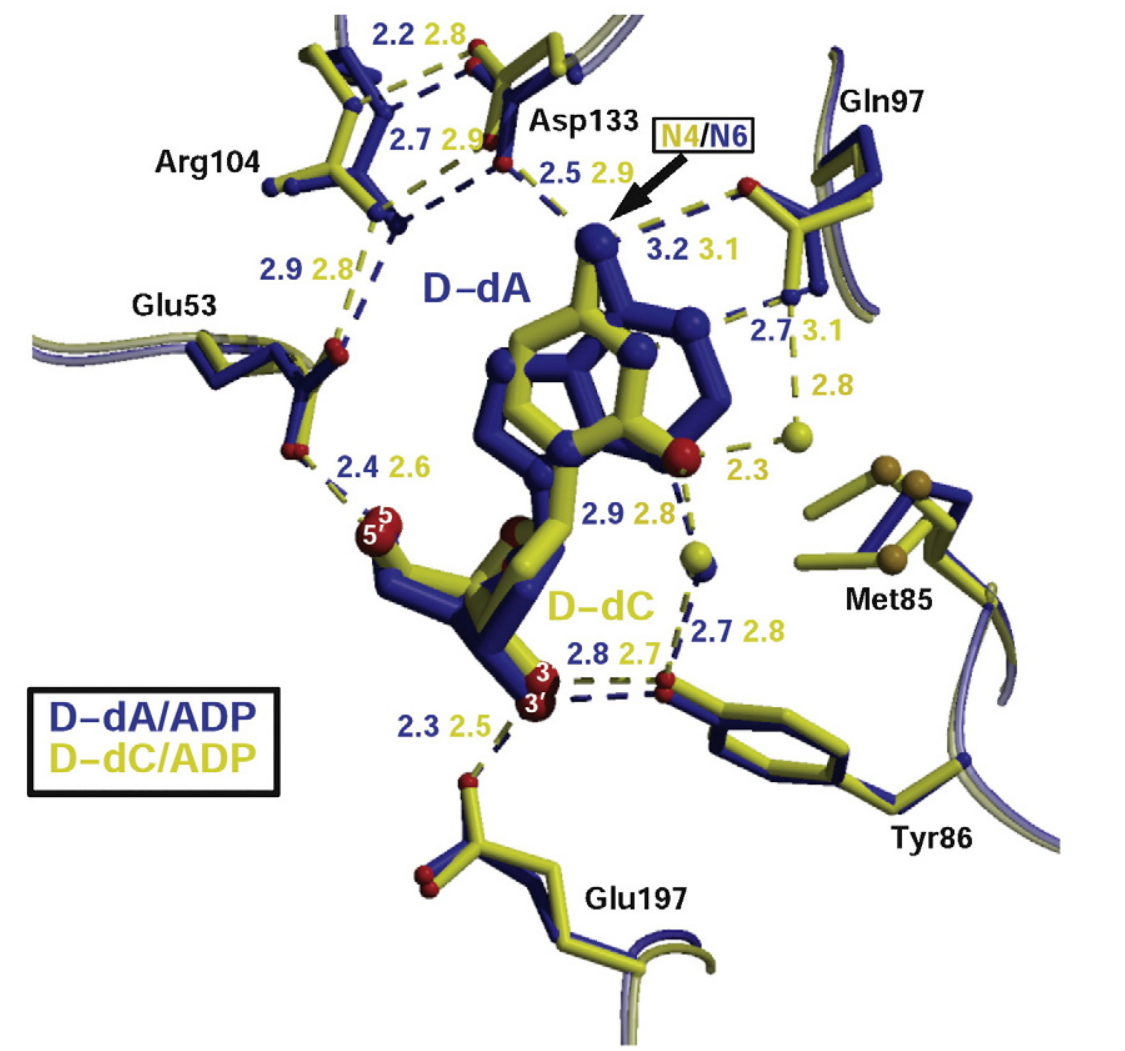

Fig. 1. Binding of the purine $\mathrm{dA}$ relative to the pyrimidine $\mathrm{dC}$ requires only a slight adjustment of active site residues. (a) A stereoview of the overlay of the D-dA/ADP complex (blue) on the D-dC/ADP complex (yellow; PDB ID 1P60). Note the nearly perfect overlay of the protein, and of the nucleosides/nucleotides. (b) The larger adenine ring of dA induces movement in several active site residues relative to their position with $\mathrm{dC}$. This permits the maintenance of enzymenucleoside interactions. Importantly, the side chain of Glu53 maintains its interaction with the D-dA 5'-OH group. The short distances between Asp133 to Arg104, and Glu197 to the 3'-OH group were verified by inspection of electron density maps. Met85 in the D-dC structure exhibited two conformations; both are shown. All structure figures were prepared using MOLSCRIPT ${ }^{19}$ or Bobscript, ${ }^{20}$ and rendered with Raster3D. ${ }^{21}$

of $0.89 \AA$ on $206 \mathrm{C}_{\alpha}$ atoms. The main differences in conformations that the enzyme assumes in the two complexes are predominantly confined to three sections (labeled A, B and C in Fig. 2a) that, while discontinuous in their sequence, together build a continuous surface.

We propose that the three distinct areas A, B and C communicate between each other upon binding of either ADP or UDP, and that this ultimately affects the conformation of the nucleoside. To illustrate how this communication may take place, we will start by analyzing the structural differences that occur in region $\mathrm{A}$, the region involved in the binding of the base moiety of the nucleotides. We observe that this region senses the difference between ADP and UDP, and accordingly adjusts the conformation of the loop that directly interacts with the base moiety of the donor (arrow 1, base-sensing loop in Fig. 2a). A similar conformational change for this loop occurring upon binding of UDP, described by Godsey et al. ${ }^{7}$ was observed in the structural examination of a $\mathrm{dCK}$ variant lacking the insert region (the insert region is a component of region $\mathrm{C}$ ). The fact that we also observe this same main-chain reorganization in response to the nature of the phosphoryl donor, using a construct of full-length $\mathrm{dCK}$, verifies this conformational response to the nature of the phosphoryl donor. The change in conformation of 
(a)

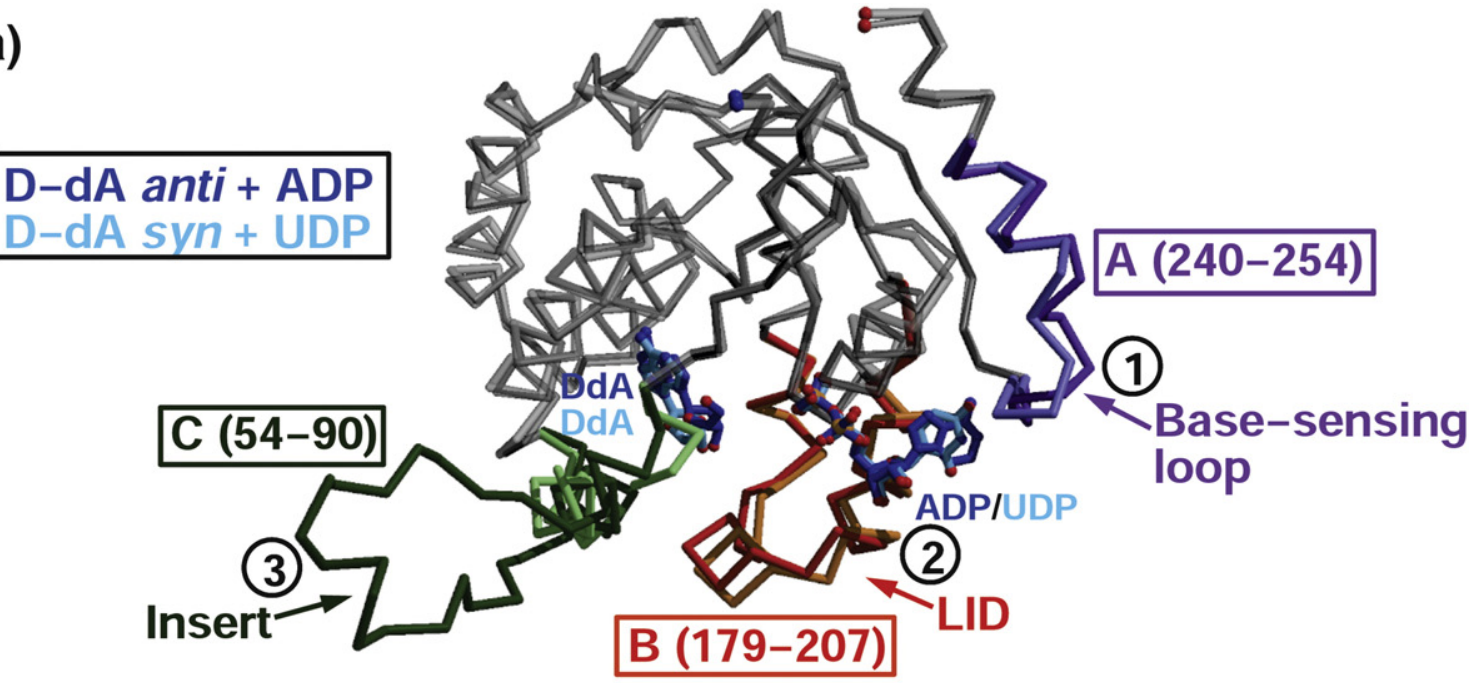

(b)
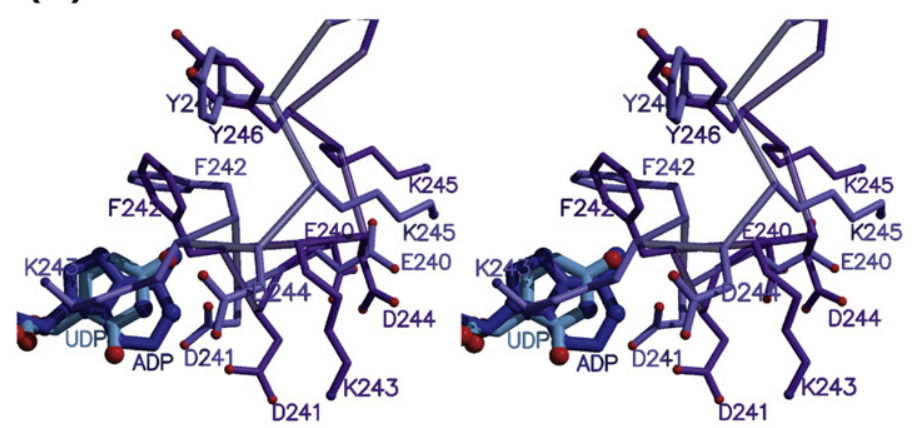

(d)

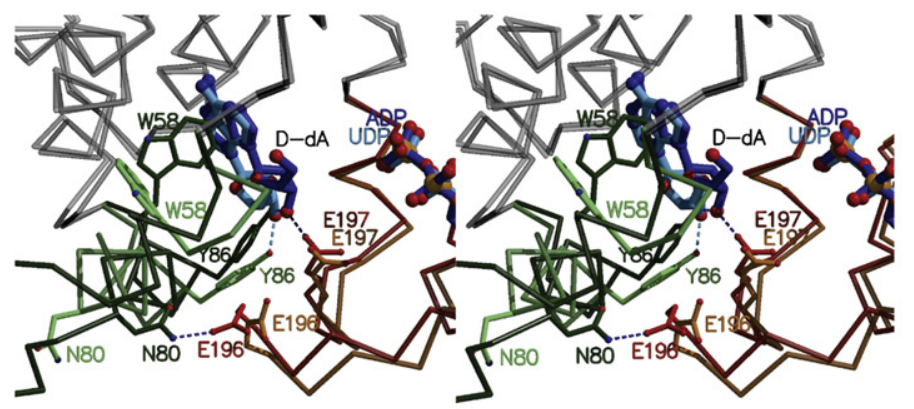

(c)

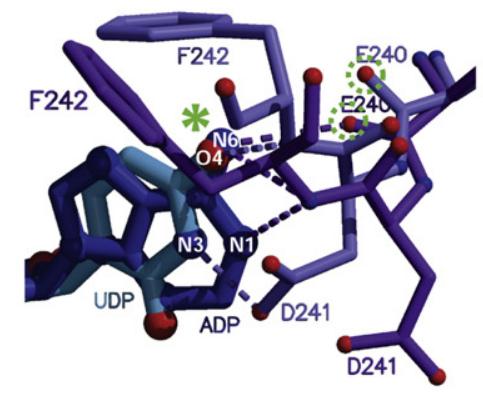

(e)

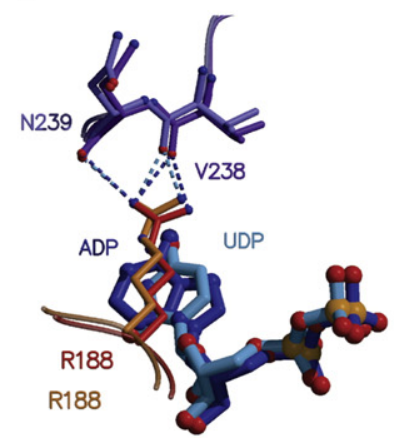

Fig. 2. Binding of UDP instead of ADP results in conformational changes limited to three regions. (a) Overlay of the $\mathrm{D}-\mathrm{dA} / \mathrm{ADP}$ and the D-dA/UDP structures. Regions that overlay well are colored gray. The three regions that differ in conformation between the two complexes are region A (ADP complex in dark purple, UDP complex in light purple), region B (ADP complex in red, UDP complex in yellow), and region C (ADP complex in dark green, UDP complex in light green). Arrows point at the important motifs that belong to each region: Insert, LID, and Base-sensing loop. The nucleoside/nucleotide is shown in dark blue for the ADP complex, and in light blue for the UDP complex. The coloring scheme in panel a is used in all panels. (b) A stereoview of the base-sensing loop. Note the complete reorganization of this loop according to the nature of the diphosphate. (c) Close-up of the base (adenine or uracil) - enzyme interaction. The amino group of ADP overlays on the carbonyl group of UDP (marked with an asterisk $\left(^{*}\right)$ ). The different hydrogen bonding properties of these moieties induce the adjustment in enzyme conformation. The carbonyl group of Glu240 (circled in green), which interacts with the amino group in the ADP structure, is forced to increase its distance to the UDP carbonyl group. (d) A stereoview of the region B - region C interface. In the open UDP structure, Glu196 cannot maintain its interaction with Asn 80 as seen in the ADP complex. Note also the different conformations of Trp58 and Tyr86 in region C. (e) Close-up of the stacking interaction between the guanidinium group of Arg188 and the base moiety of ADP or UDP. Positioning of Arg188 may determine the conformation of the enzyme, since this residue acts to line regions $\mathrm{A}$ and $\mathrm{B}$. 
the loop that directly interacts with the base (for details see Fig. $2 b$ ) extends to the whole region that we identify as region A (residues 240-254; the ADP complex in dark purple and the UDP complex in light purple).

What is the structural feature that discriminates a uracil base from an adenine moiety? With ADP, the amino group (N6) interacts $(3.2 \AA)$ with the mainchain carbonyl group of Glu240, while nitrogen N1 accepts an H-bond from the main-chain -NH- of Phe242 (Fig. 2c). Upon binding of UDP, the carbonyl group in position 4 of the uracil base binds in the same position as the amino group (N6) of the adenine ring (labeled with an asterisk $\left({ }^{*}\right)$ in Fig. 2c). If dCK would maintain its ADP-bound conformation, an unfavorable interaction between the uracil carbonyl group and the Glu240 carbonyl group would take place (the position of the carbonyl group of Glu240 in the ADP and UDP structures is marked by green circles in Fig. 2c). This is possibly the driving force for reorganization of the base-sensing loop. The carbonyl group of Glu240 increases its distance to the uracil base by reorganizing Phe242 and attracting the side chain of Asp241 closer to the uracil ring. This movement allows for the formation of a stabilizing hydrogen bond between N3 of the uracil base and the carboxylic group of Asp241 (Fig. 2c). To recapitulate, the base-sensing loop adopts a main-chain conformation that varies according to the nature of the nucleotides, resulting in different enzymenucleotide base interactions taking place.

A second area that changes conformation due to the presence of UDP versus ADP is region B (residues 179-207 in red and yellow, Fig. 2d). This region encompasses the LID motif (arrow 2 in Fig. 2a), an important motif in nucleoside kinases that contains several arginine residues that take part in nucleotide donor binding and in transition state stabilization. How does region B sense the nature of the phosphoryl donor (ADP versus UDP)? Inspection of the structures does not provide a clear communication pathway connecting the base-sensing loop in region A to region $\mathrm{B}$. One possible information flow involves Arg188 that acts to directly connect the LID motif with the base-sensing loop (Fig. 2e). However, due to the relatively small change in position for the atoms involved in this interaction (about $0.4 \AA$ ), this model for communication between regions $A$ and $B$ must be validated by further experiments. An alternative explanation for the difference in conformation of region $B$ involves the direct sensing of the nature of the donor by the LID arginine Arg188. The guanidinium group of Arg188 makes a $\pi-\pi$ interaction with either the adenine or uracil base of the nucleotides. Since the respective base moieties are somewhat displaced relative to each other, the position of the side chain of Arg188 adjusts accordingly, and this adjustment propagates to the entire region B (Fig. 2e).

The third region that varies significantly in conformation between the ADP and UDP structures is region $C$ (arrow 3 in Fig. 2a). The insert sequence is a component of region $C$. The insert sequence extends in dCK from residue 63 to residue 77 . The role of the insert in $\mathrm{dCK}$ is still not clear but our studies of a dCK variant lacking this insert ${ }^{7}$ have shown a change in the selectivity of the enzyme between pyrimidine and purine substrates. In all the ADP-containing dCK structures solved so far where the insert was traceable (attributed to stabilization of the insert through crystal packing), this region adopts the same conformation. In the UDP-containing structure presented here there is no clear electron density for residues 59-79. However, it is evident from those modeled residues flanking the insert region that in the presence of UDP the insert region adopts an entirely different conformation from the one modeled in the presence of ADP. It is noteworthy that the residues that move the most between the ADP and the UDP structures are located just before and just after the insert region: Trp58, Asn80, and Tyr86 (Fig. 2d).

To reiterate, the presence of UDP or ADP, first sensed by region $A$, influences the conformation of region $\mathrm{B}$ to become more open, which in turn determines the conformation of region $\mathrm{C}$. Residues from regions $\mathrm{B}$ and $\mathrm{C}$ interact directly with the nucleoside. In the ADP complex, the closed conformation of the LID (region B) allows a direct interaction with region C (i.e., Glu196 with Asn80, Fig. 2d). In contrast, due to the open LID conformation in the UDP complex, this interaction connecting region $B$ to region $C$ is not possible. In fact, Asn80, a residue flanking the insert region, adopts a very different conformation between the two complexes (Fig. 2d). Importantly, in the closed LID conformation (i.e., with $\mathrm{ADP})$, a direct interaction $(2.3 \AA)$ between the LID residue Glu197 and the $3^{\prime}-\mathrm{OH}$ group of D-dA takes place, thereby stabilizing the closed state. Such an interaction is not possible in the presence of UDP (the distance between Glu197 and the $3^{\prime}-\mathrm{OH}$ increases to $5.2 \AA$; Fig. 3a). To summarize this section, $\mathrm{dCK}$ has the ability to sense the nature of the nucleotide at the donor site, and when bound to UDP instead of ADP, the enzyme adopts a more open conformation in regions $\mathrm{A}, \mathrm{B}$, and $\mathrm{C}$.

\section{The identity of the nucleotide bound at the donor site affects the conformation of the nucleoside at the acceptor site}

In addition to the changes in $\mathrm{dCK}$ conformation in regions $\mathrm{A}, \mathrm{B}$ and $\mathrm{C}$ discussed above, superposition of the structures solved in the presence of ADP versus UDP reveals a striking difference in the conformation of the nucleoside D-dA. In the ADP complex, D-dA binds in the anti conformation. This allows residues Tyr86 and Glu197 to hydrogen bond with the 3'-OH group of the nucleoside (Fig. 3a). We designate this conformation as active because in this conformation the $5^{\prime}-\mathrm{OH}$ group of $\mathrm{D}-\mathrm{dA}$ is within hydrogen bonding distance to Glu53 (2.4 A, Fig. 3a). This closed conformation allows Glu53 to act as a base that activates the $5^{\prime}-\mathrm{OH}$ for attack on the $\gamma$ phosphate group of the phosphoryl donor. This is 
the typical conformation adopted by all the pyrimidine + ADP structures that we have solved so far. $^{3-5}$

(a)

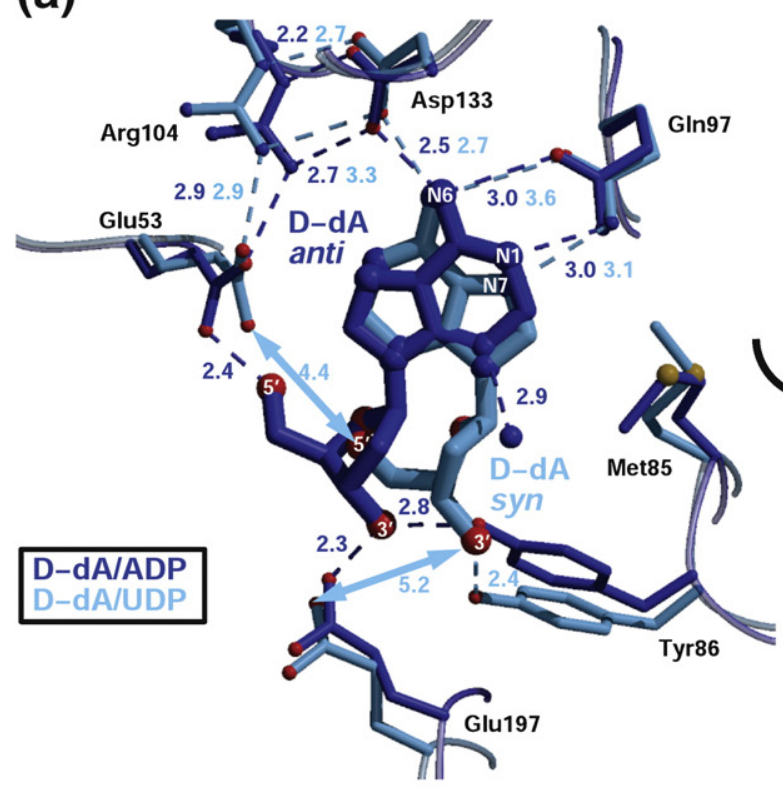

(c)

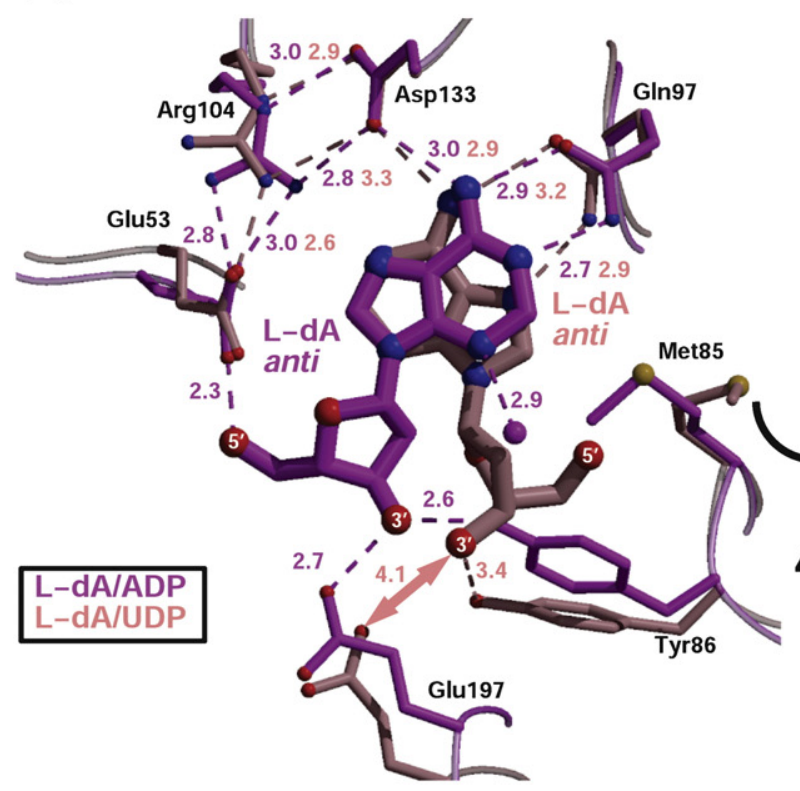

In contrast, in the D-dA complex with UDP, the nucleoside binds in the unexpected syn conformation. The base moiety of the D-dA bound in syn (b)

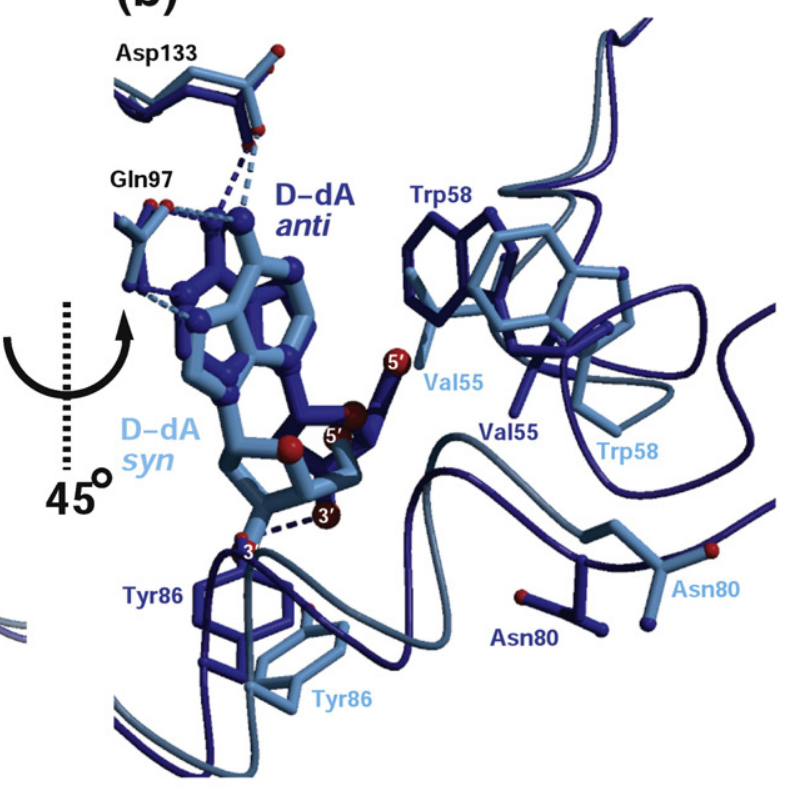

(d)

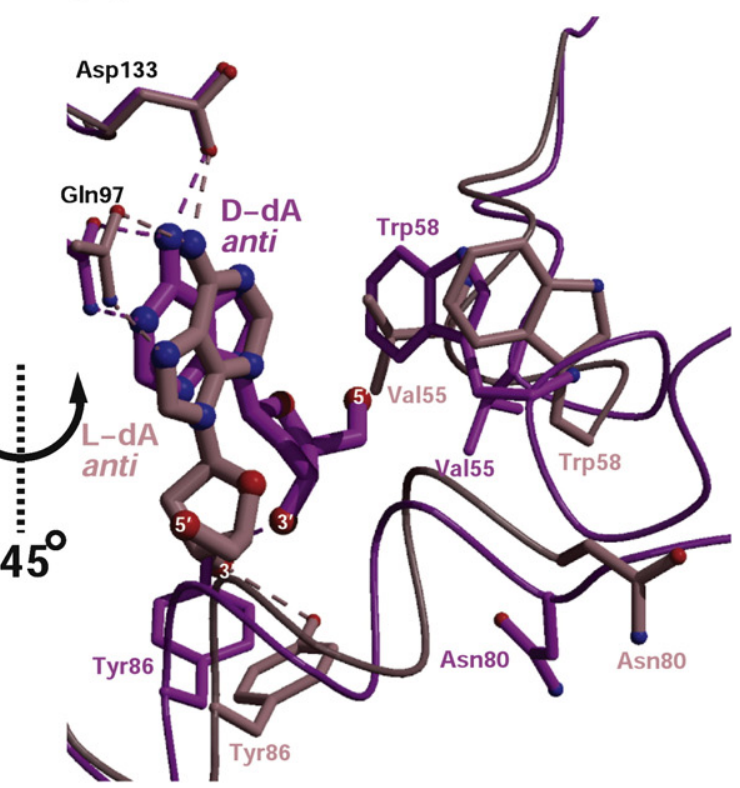

Fig. 3. The base moiety of D- and L-dA occupy the same region regardless of the nature of the nucleotide at the donor site, whereas the position of the sugar component of the nucleoside is determined by the nucleotide. (a) An overlay of the nucleoside and active site residues of the D-dA/ADP (dark blue) and D-dA/UDP (light blue) structures. Hydrogen bond interactions are marked by broken lines in the corresponding colors, with the distance given in $\AA$. Interactions that are absent in the UDP complex are detailed by thick light blue arrows. The D-dA binds in the anti conformation when ADP is at the donor site, but in the syn conformation when UDP is at the donor site. Despite the different nucleoside conformation, the base moiety occupies the same space and the interactions to the enzyme are recapitulated in both cases. In contrast, the sugar moiety of D-dA in the presence of UDP is such that the interactions to Glu53 (via the $5^{\prime}-\mathrm{OH}$ ) and Glu197 (via the 3'-OH) are lost. Therefore, we designate this conformation as inactive. See the text for details. (b) A $\sim 45^{\circ}$ rotation relative to the view in panel a. In this view, the large change in conformation of Trp58 is apparent. Asp133 is used in these and subsequent figures as a common reference point. (c) An overlay of the nucleoside and active site residues of the L-dA/ADP (dark pink) and D-dA/UDP (light pink) structures. Here too, the adenine portion of the nucleoside overlays well, despite the different relative orientation (both are in the anti conformation). The sugars of L-dA are in a significantly different position, so that the important interactions to Glu53 and Glu197 cannot be made in the complex with UDP. (d) A $\sim 45^{\circ}$ rotation relative to the view in (c). 
(UDP complex) is turned $180^{\circ}$ relative to the base in the ADP complex that is in anti. Nevertheless, the adenine ring preserves the same hydrogen-bonding pattern with the enzyme regardless of being in syn or anti. The adenine amino group is at the same position in both the syn and anti conformations, preserving its interaction with Asp133. On the other hand, the interaction with the side chain of Gln97 is via N1 when the base is in anti (ADP complex), and is replaced by N7 when the base is in syn (UDP complex) (Fig. 3a). While the adenine moieties of $\mathrm{D}-\mathrm{dA}$ occupy the same binding site regardless of their conformation (syn or anti), the sugar moieties of the nucleosides occupy entirely different positions. The $5^{\prime}-\mathrm{OH}$ group of syn $\mathrm{D}-\mathrm{dA}$ is $4.4 \AA$ away from Glu53 while it is $2.4 \AA$ away in the anti D-dA. Such a large separation between Glu53 and the $5^{\prime}-\mathrm{OH}$ suggests that the state we have trapped within the D-dA/UDP structure would be catalytically inactive.

How can we rationalize this observation where the enzyme conformation is not compatible with catalysis? In fact, kinetic experiments show that dCK is active with UTP as phosphoryl donor (Table 2). Thus, we interpret the observed enzyme conformation as an intermediate state during the catalytic cycle. The fact that we were able to trap such a state for the UDP complex, but not for the ADP complex, may be related to the different mechanism of action of dCK when it uses UTP versus ATP. ${ }^{8,9}$ It is not clear if the enzyme also goes through such a state in the presence of ATP, but fluorescence experiments are consistent with dCK adopting a different conformation upon UTP binding versus that adopted upon ATP binding (see below). Moreover, the different kinetic parameters measured in the presence of ATP or UTP as phosphoryl donors are consistent with the fact that we observed different states for the complexes with ADP to those with UDP.

In the D-dA/UDP complex, the adoption of the syn conformation by $\mathrm{D}-\mathrm{dA}$ and its rotation by $180^{\circ}$ compared to its conformation in the ADP complex is accompanied by adjustment in the position of neighboring residues. Most notably are changes in Val55, Trp58, Tyr86, and Glu197 (Fig. 3). In the D-dA/ADP complex, Trp58 and the adenine ring are perpendicular to each other, while in the D-dA/ UDP structure, Trp58 rotates away from the nucleoside and is at $\sim 45^{\circ}$ to the base of $\mathrm{dA}$ (Fig.

Table 2. Kinetic analysis of $\mathrm{C}_{4} \mathrm{~S}-\mathrm{dCK}$ and WT-dCK using ATP or UTP as donors and D-dA or L-dA as acceptors

\begin{tabular}{lcccc}
\hline Substrates & Enzyme & $k_{\text {cat }}$ & $\mathrm{Km}(\mathrm{D} / \mathrm{L}-\mathrm{dA})$ & $k_{\text {cat }} / \mathrm{Km}\left(\times 10^{-3}\right)$ \\
\hline D-dA+ ATP & WT & 2.13 & 115 & 18.5 \\
& $\mathrm{C}_{4} \mathrm{~S}$ & 1.85 & 311 & 5.9 \\
L-dA+ ATP & WT & 0.92 & 110 & 8.4 \\
& $\mathrm{C}_{4} \mathrm{~S}$ & 0.84 & 190 & 4.4 \\
D-dA+ UTP & WT & 0.37 & 8 & 46 \\
& $\mathrm{C}_{4} \mathrm{~S}$ & 0.24 & 44 & 5.5 \\
L-dA+ UTP & WT & 0.24 & $<5$ & $>48.0$ \\
& $\mathrm{C}_{4} \mathrm{~S}$ & 0.31 & 16 & 19.4 \\
\hline
\end{tabular}

Values shown are the averages of at least two experiments. $k$ cat are in $\mathrm{s}^{-1}, \mathrm{Km}$ are in $\mu \mathrm{M}$, and $k \mathrm{cat} / \mathrm{Km}$ are in $\mu \mathrm{M}^{-1} \mathrm{~s}^{-1}$. 3b). Val55 moves accordingly, always facing Trp58. Despite these considerable conformational changes, the catalytically important Glu53 maintains the same position, being anchored by the preceding and following proline residues (Pro52 and Pro54). This change in conformation of the nucleoside and its adjacent active site residues, depending on the nature of the donor, ADP versus UDP, is recapitulated in the complexes where $\mathrm{L}-\mathrm{dA}$ is present instead of D-dA (see below and Fig. $3 c$ and d).

\section{The L- versus the D-enantiomer of $\mathrm{dA}$}

The above discussion compared complexes of $\mathrm{dCK}$ that differ either in the nature of the nucleoside acceptor (D-dC versus D-dA, both with ADP) or in the nature of the nucleotide donor (ADP versus UDP, both with D-dA). An additional point we set out to delineate is the structural properties that allow dCK to phosphorylate both physiological (D-form) and non-physiological (L-form) nucleosides. The enzyme $\mathrm{dCK}$ is rather unique in not being enantioselective. Addressing this question has medicinal implications as several antiviral nucleoside analogs possess the L-chirality, and are dependent on $\mathrm{dCK}$ for their pharmacological activity.

In our previous work we addressed this question in the case of pyrimidine substrates with the Lchirality, e.g. L-dC and lamivudine. ${ }^{4,5}$ To reveal if the same conformational adjustments as seen for Lpyrimidine substrates take place with purines, we solved the structure of dCK in complex with L-dA, with either ADP or UDP at the donor site.

\section{Comparison of the D-dA/ADP to L-dA/ADP structure}

In the ADP complex, $\mathrm{L}-\mathrm{dA}$ binds in the active site of $\mathrm{dCK}$ in a very similar way compared to $\mathrm{L}-\mathrm{dC}$ (Fig. 4), the only main difference being the positioning of residue Gln97, which moves further away from the active site in order to make space for the larger adenine ring. Additionally, the water molecule formerly bridging between the carbonyl group of the cytosine base and Gln97 gets displaced in the $\mathrm{L}-\mathrm{dA}$ structures. However, the water molecule bridging the adenine ring and Tyr86 is retained. This scenario is very similar to that discussed previously for the D-dC and D-dA couple (Fig. 1b).

Comparison of the protein conformation between the D-dA and L-dA structures solved in the presence of ADP are somewhat complicated by the fact that the enzyme crystallized in different space groups. The D-dA/ADP complex was solved in an orthorhombic space group while L-dA/ADP in a tetragonal space group. The main difference between the two space groups is that in the tetragonal space group, due to crystal packing, Arg192 points away from the ADP molecule. This is a common feature that has been observed so far for all the dCK structures solved in the tetragonal space group (PDB codes 1P5Z, 1P61, and 1P62). We note this because some of the differences between D-dA/ADP and L-dA/ 


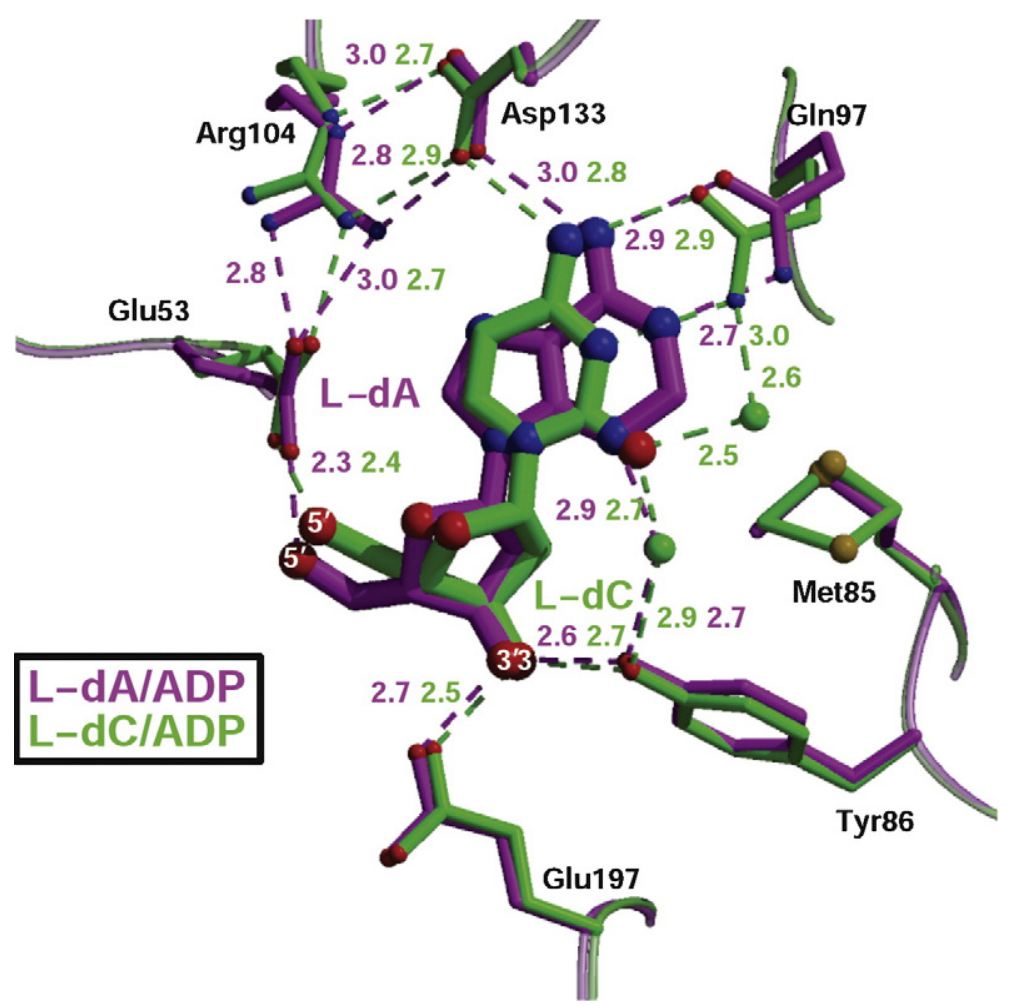

Fig. 4. Binding mode of $\mathrm{L}-\mathrm{dA}$ resembles that of $\mathrm{L}-\mathrm{dC}$. The overall fold of the enzyme is maintained (a closed conformation), and active site residues need only to adjust slightly to accommodate the large purine ring. Importantly, the water molecule bridging the base of the nucleoside (either via the carbonyl of L-dC, or nitrogen N3 of L-dA) and Tyr86 is retained, supporting the enzyme adopting the closed and active conformation. Met85 in the L-dC structure exhibited two conformations; both are shown.

ADP are actually caused by the change in space group. Thus, a component of the rmsd $(0.64 \stackrel{\mathrm{A}}{\text { on }}$ 226 atoms) between the D-dA/ADP and the L-dA/ ADP can be attributed to crystal packing, and not to the difference in chirality of the nucleoside acceptor. In other words, when taking into consideration differences due to crystal packing, the conformation of $\mathrm{dCK}$ between the D-dA/ADP and the L-dA/ADP structures is very similar. That is, the enzyme adopts the closed conformation for regions $\mathrm{A}, \mathrm{B}$, and $\mathrm{C}$ in both complexes.

Concerning the conformation of the nucleoside $\mathrm{dA}$, the adenine rings of the $\mathrm{D}$ - and L-enantiomers overlay quite well. This permits the preservation, regardless of the chirality of $\mathrm{dA}$, of similar hydrogen bonding interactions to active site residues (Fig. 5a). By tilting the base of $\mathrm{L}-\mathrm{dA}$ compared to that of $\mathrm{D}-\mathrm{dA}$, the enzyme is able to position the $3^{\prime}-$ and $5^{\prime}-\mathrm{OH}$ groups of $\mathrm{L}-\mathrm{dA}$ in the same positions as those adopted by D-dA (Fig. 5b). Such relative base tilting was also observed between the $\mathrm{D}-\mathrm{dC}$ and $\mathrm{L}-\mathrm{dC}$ complexes. ${ }^{5}$ This tilting is significant, as it allows the 5 -OH group to maintain its hydrogen bonding distance to Glu53 (Fig. 5a).

\section{Comparison of the D-dA/UDP to L-dA/UDP structure}

The results presented earlier showed that in the case of the nucleoside $\mathrm{D}-\mathrm{dA}$ the conformation of
$\mathrm{dCK}$ is dependent on the nature of the nucleotide at the donor site (ADP versus UDP). This raised the question of whether this phenomenon would also occur with the L-form of the nucleoside, or is it specific for D-dA. To address this, we solved the structure of dCK in complex with L-dA and UDP. Comparison of the L-dA/UDP and the D-dA/UDP complex structures reveals a nearly identical enzyme conformation (rmsd $0.45 \AA$ on 213 atoms). In both complexes the enzyme adopts the open/ inactive conformation, as defined by the Glu53 distance from the $5^{\prime}-\mathrm{OH}$ group. This shows that the differential conformation of $\mathrm{dCK}$ in response to the nature of the nucleotide at the donor site (e.g. ADP versus $\mathrm{UDP}$ ) is not limited to $\mathrm{D}-\mathrm{dA}$, and may be a general property of the enzyme.

However, in contrast to the same protein conformation, the nucleosides, L- versus D-dA, bind differently depending on their chirality. With UDP in the donor site, D-dA binds in syn while L-dA binds in anti. Despite their different conformation, the adenine bases of the two enantiomers overlap almost perfectly and maintain the same hydrogen bonding pattern with Asp133 and Gln97 (Fig. 5c). This is observed also for the same enantiomeric couple in complex with ADP (Fig. 5a). However, since L-dA binds in anti, the deoxyribose sugar is positioned in a mirror fashion to the sugar of $\mathrm{D}-\mathrm{dA}$ (Fig. 5c). Interestingly, this still allows the $3^{\prime}-\mathrm{OH}$ group of L-dA to occupy a comparable position to 
(a)

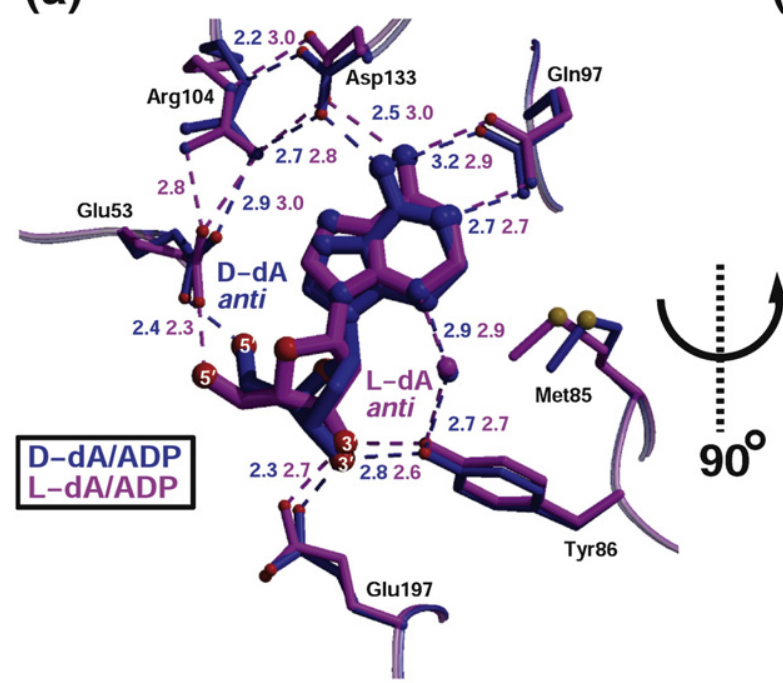

(b)

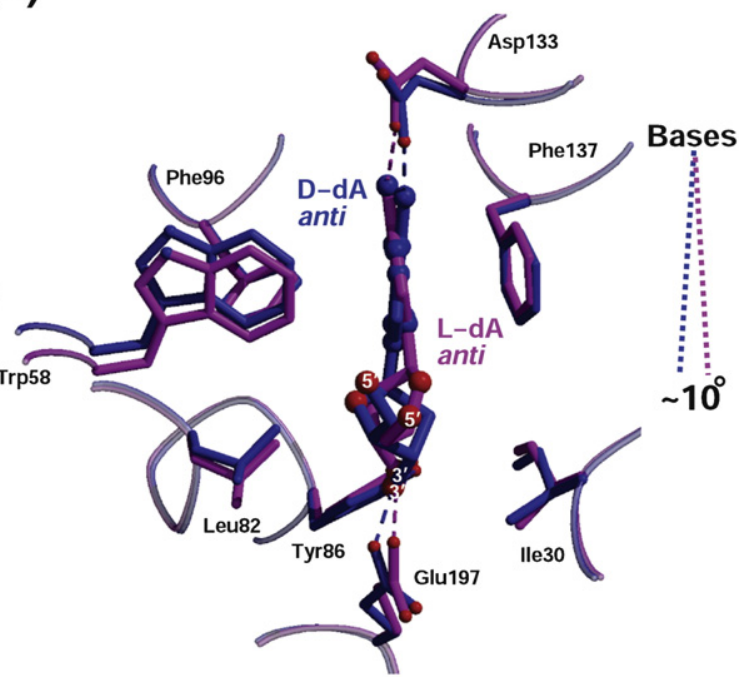

(c)

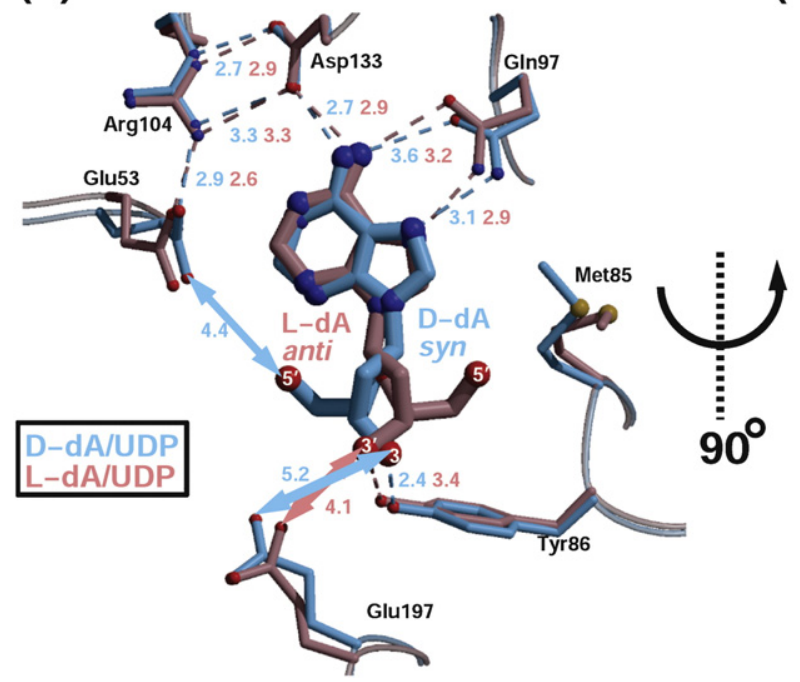

(d)

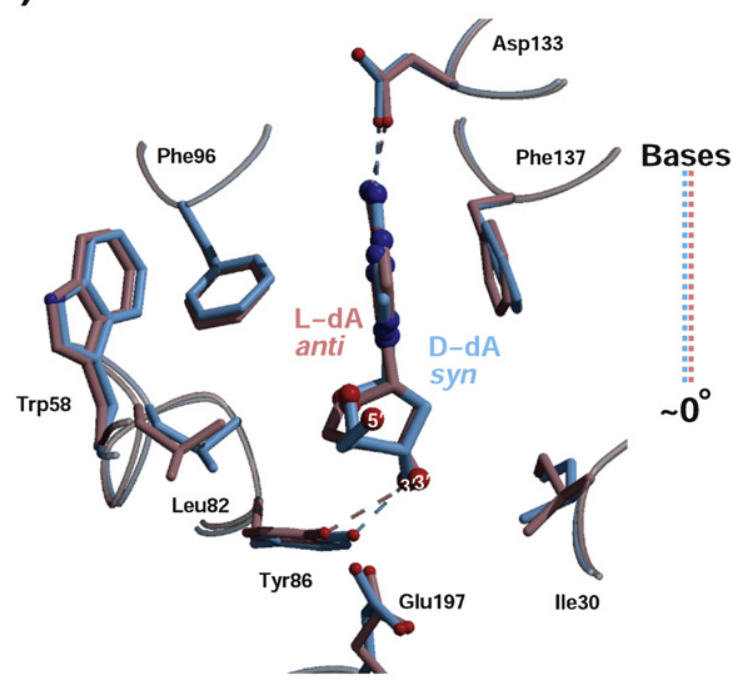

Fig. 5. Regardless of the chirality of the nucleoside, dCK adopts an active conformation if ADP is present at the donor site, and an inactive conformation if UDP is present at the donor site. (a) An overlay of the D-dA/ADP (dark blue) and L-dA/ADP (dark pink) structures shows a very similar enzyme (closed) and nucleoside (anti) conformation. (b) A $\sim 90^{\circ}$ rotation relative to the view in panel a. The binding of the L-enantiomer is characterized by a base tilt of $\sim 10^{\circ}$ relative to its position in the D-enantiomer (see the schematic at the right). (c) An overlay of the D-dA/UDP (light blue) and L-dA/UDP (light pink) structures shows a very similar enzyme conformation (open). In both cases the enzyme cannot make the necessary interactions via Glu53 to the 5'-OH group of the nucleoside. In the D-dA complex the orientation of the sugar is correct, but the distance is too large $(4.4 \AA)$. In the L-dA complex, the nucleoside is in the anti conformation, but the sugar is pointing in the opposite direction (towards Met85) to what would allow Glu53 to activate it. Thus, in both complexes of UDP with L-dA or D-dA, the enzyme conformation represents an inactive state. (d) A $\sim 90^{\circ}$ rotation relative to the view in (a). In the structures with UDP, both bases adopt the same tilt (see the schematic at the right). Note how Trp58 in the UDP family of structures turns by $45^{\circ}$ away from the adenine base of dA compared to the ADP family of structures (see (b) use Phe96 as a reference) and how Tyr86 and Glu197 move down (this movement is visible also in Fig. 3).

the $3^{\prime}-\mathrm{OH}$ of $\mathrm{D}-\mathrm{dA}$. In contrast, the $5^{\prime}-\mathrm{OH}$ faces towards Met85 instead of Glu53, as was seen for $\mathrm{D}-\mathrm{dA}$. This corresponds to an inactive conformation, albeit different from that seen in the D-dA/UDP complex. In the latter, while the $5^{\prime}-\mathrm{OH}$ group is too far from Glu53 for this side chain to activate the hydroxyl group for attack on the phosphoryl donor (4.4 $\AA$ ), the 5'-OH still faces Glu53 (Fig. 5c).

As was argued for the case of D-dA and UTP as donor, we interpret this inactive enzyme conforma- tion to represent an intermediate state, and that such a state can easily transition to an active state. In fact, kinetic analysis shows that L-dA has similar $k_{\text {cat }}$ and $K_{\mathrm{m}}$ values to D-dA when using UTP as phosphoryl donor (Table 2). This suggests that for both cases the enzyme can efficiently transition from the inactive states we observe to a state that is catalytically competent. At the same time, the kinetics for both of the nucleosides D-dA and L-dA show a higher $k_{\text {cat }}$ with ATP, consistent with the fact that with this 
phosphoryl donor the enzyme avoids the inactive conformation we see with UDP. In contrast to a higher $k_{\text {cat }}$ with ATP, the $K_{\mathrm{m}}$ values for the nucleosides is lower with UTP.

As mentioned above, in the complexes with ADP the base of L-dA tilts relative to the position of the base of D-dA to allow productive binding of the nonphysiological enantiomer of dA (Fig. 5b). In contrast, in the complexes with UDP, no tilting occurs between the enantiomeric couple of $\mathrm{dA}$ (Fig. 5d).

\section{Fluorescence experiments are consistent with phosphoryl donor-dependent enzyme states}

The above structural results revealed a closed $\mathrm{dCK}$ conformation in the presence of ADP, and an open state in the presence of UDP. To verify that this phenomenon is not an artifact of the crystalline state or a unique feature of the $\mathrm{C}_{4} \mathrm{~S}-\mathrm{dCK}$ mutant used for the structural studies, and to test if the differential states observed with the nucleotide diphosphates are relevant to the true triphosphate substrates, we used intrinsic tryptophan fluorescence to detect nucleotide-dependent conformational changes in $\mathrm{dCK}$.

The tryptophan emission intensity of $\mathrm{C}_{4} \mathrm{~S}-\mathrm{dCK}$ does not change significantly upon addition of ATP. On the other hand, addition of UTP results in a $\sim 8 \%$ increase in the tryptophan fluorescence signal (Fig. 6). Our structural studies revealed that Trp58 changes its conformation dramatically between the complexes solved in the presence of ADP to those solved in the presence of UDP. Thus, the change in tryptophan fluorescence upon addition of UTP can be rationalized by the conformational change in Trp58 as seen in our structures. On the other hand, the lack of change in tryptophan fluorescence upon addition of ATP suggests that the apo form of dCK and the ATP-bound form have a very similar conformation. The fact that similar changes in tryptophan signal are observed when the diphosphates (Supplementary Data Fig. S2) are titrated instead of the triphosphates (Fig. 6) suggest that the structural data, obtained as the dead-end complexes with a nucleoside at the acceptor site (D- or L-dA) and a diphosphate at the donor site (ADP or UDP), do indeed describe the conformational changes that occur in $\mathrm{dCK}$ upon the productive binding of substrates. As a further control, to assess the relevance of the results obtained with the $\mathrm{C}_{4} \mathrm{~S}$ variant of $\mathrm{dCK}$ to that of the wild type enzyme, we tested the behavior of WT-dCK in its response to ADP or UDP binding. Our results show an identical change in fluorescence of WT-dCK to that of the $\mathrm{C}_{4} \mathrm{~S}$ variant (Supplementary Data Fig. S3). Consistent with both our structural and our tryptophan fluorescence data are results from the Eriksson group that also detected, by fluorescence, a change in $\mathrm{dCK}$ conformation due to substrate binding. ${ }^{10,11}$ In earlier work, pre-steady state experiments suggested that ATP and UTP bind differently to the enzyme. ${ }^{10}$ In more recent work, using dCK labeled with fluorescent probes at various cysteine residues,

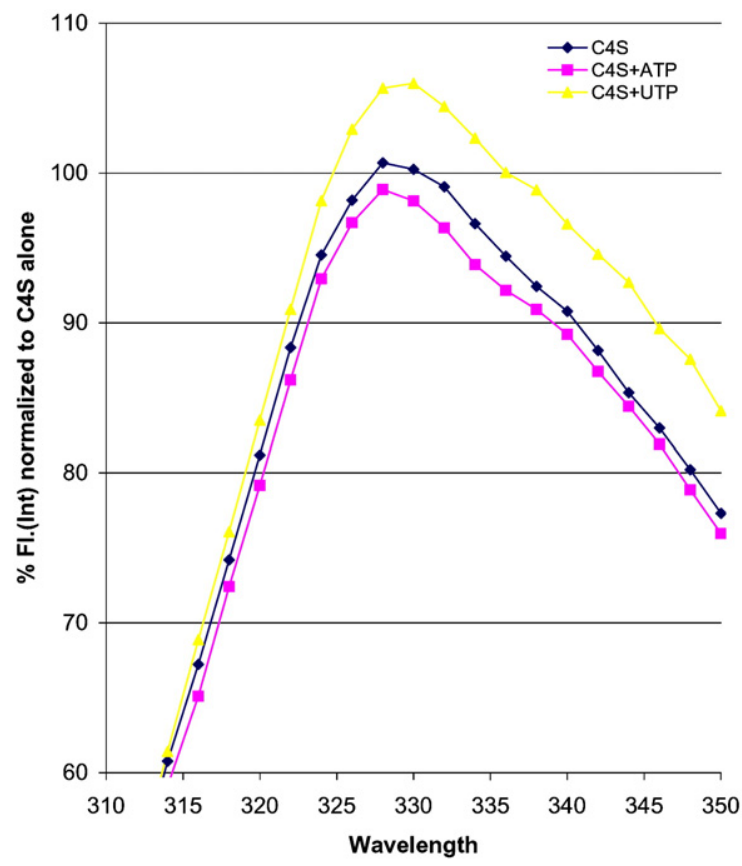

Fig. 6. Tryptophan fluorescence emission scan reveals a change in tryptophan signal upon addition of UTP but no change with ATP. Spectra were recorded for the protein alone (black), protein with ATP (magenta), and protein with UTP (yellow). The presence of UTP results in $\sim 8 \%$ increased tryptophan signal.

the researchers determined that the sensitive cysteine residue in dCK was Cys185. ${ }^{11}$ This conclusion is consistent with our structural results that demonstrate a movement in region $B$, which includes this residue. In addition, it was concluded that the loss in energy transfer in these experiments was due to movement in Trp58. Our results demonstrate a rare case in which a change in tryptophan fluorescence seen upon ligand binding can be correlated directly with a structural change of a specific tryptophan residue.

\section{The open/catalytically incompetent state is an intermediate within the dCK catalytic cycle}

What supports our interpretation of the open/ inactive state as a true intermediate state that occurs along the reaction trajectory, versus the alternative, which is that this state is an offpathway dead-end complex? First, previous structures of $\mathrm{dCK}$ in complex with a nucleoside substrate and a nucleotide product showed a conformation that is compatible with catalysis. ${ }^{3-5,7}$ Hence, the mere combination of substrate and product does not necessarily build an inactive state, as we observe with $\mathrm{dA}$ and UDP. Second, and more important, is the fact that fluorescence experiments indicate a change in enzyme conformation upon UTP (or UDP) binding, but not with ATP (or ADP). This shows that a UTP-dependent state is adopted by $\mathrm{dCK}$ upon binding of this nucleotide. Thus, a different dCK conformation, dependent on 
the nature of the nucleotide donor, is an integral part of the $\mathrm{dCK}$ reaction trajectory. Thirdly, kinetic data have demonstrated a different kinetic behavior for this enzyme when UTP is used as a donor instead of ATP (Supplementary Data Fig. S5). ${ }^{12}$ Thus, the UDP-specific state we observe is consistent with a UDP-specific mechanism. We propose that the state we observe with UDP describes an inactive ternary complex formed after the sequential binding of both substrates (Supplementary Data Fig. S5). This inactive state must convert to the closed ternary state to allow phosphoryl transfer to occur. Due to the specific nature of $\mathrm{dA}$, we were able to trap this inactive state. With other nucleosides, e.g. dC, this open state also forms, but as the closed state is more favored, it is the one we observe in those structures. We have no indication that ATP can also induce this open enzyme state. Neither in our structural studies with ADP nor in our fluorescence experiments with ATP or ADP could we detect this open conformation.

\section{Determinants of nucleoside binding}

The change in dCK conformation between the open and closed states is limited to the regions that we defined as regions $\mathrm{A}, \mathrm{B}$, and $\mathrm{C}$. The important residues that show the most profound change are Trp58 (the source of our tryptophan signal), Asn80 (a residue that allows for region $\mathrm{B}$ - region $\mathrm{C}$ communication), and Tyr86 (Fig. 7).

Tyrosine 86 seems to be a crucial residue for the positioning of the nucleoside. Regardless of the chirality of $\mathrm{dA}$ and of the nature of the nucleotide at the donor site, this residue maintains in all four structures presented here an interaction with the $3^{\prime}-\mathrm{OH}$ group of $\mathrm{dA}$. This is achieved despite the diversity in positioning of the sugar ring of the nucleoside seen in our four structures (Fig. 7a). However, only when the base of the nucleoside interacts with Tyr86 via a water molecule does the enzyme adopt the closed (and active) state. An additional notable point is the equivalent location of the adenine base in all cases, despite a different enzyme conformation. Thus, we conclude that two factors dominate nucleoside binding: One is having the base moiety occupy the same binding site, albeit with freedom of orientation (e.g., the base can rotate $\left.180^{\circ}\right)$. Two is preserving the $3^{\prime}-\mathrm{OH}$ interaction with the Tyr86 hydroxyl group. But only in the closed state is Tyr86 positioned correctly to interact with the nucleoside via a water molecule. Additionally, only in the closed state is Glu53 able to approach the $5^{\prime}-\mathrm{OH}$ group and activate it to attack the $\gamma$-phosphate group of the donor nucleotide.

\section{Conclusion}

In addition to exposing the binding mode of $\mathrm{D}-\mathrm{dA}$, a physiological purine substrate of $\mathrm{dCK}$, our work addresses the difference in binding of L- and D-dA, and the structural ramifications of employing UTP instead of ATP as phosphoryl donor. One important conclusion that arises from this work is that dCK can adopt an open conformation with UTP, and that this conformation is catalytically incompetent. (a)

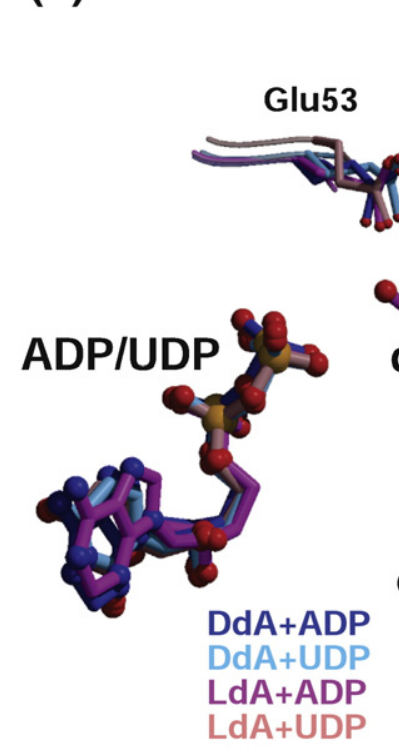

(b)

Gln97

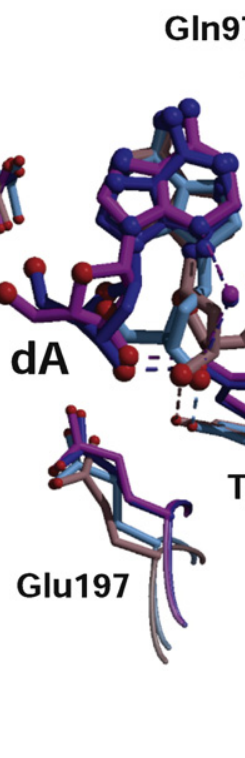

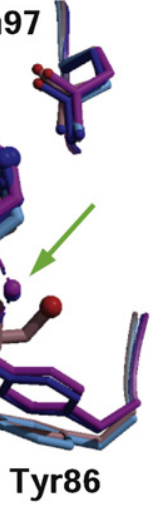

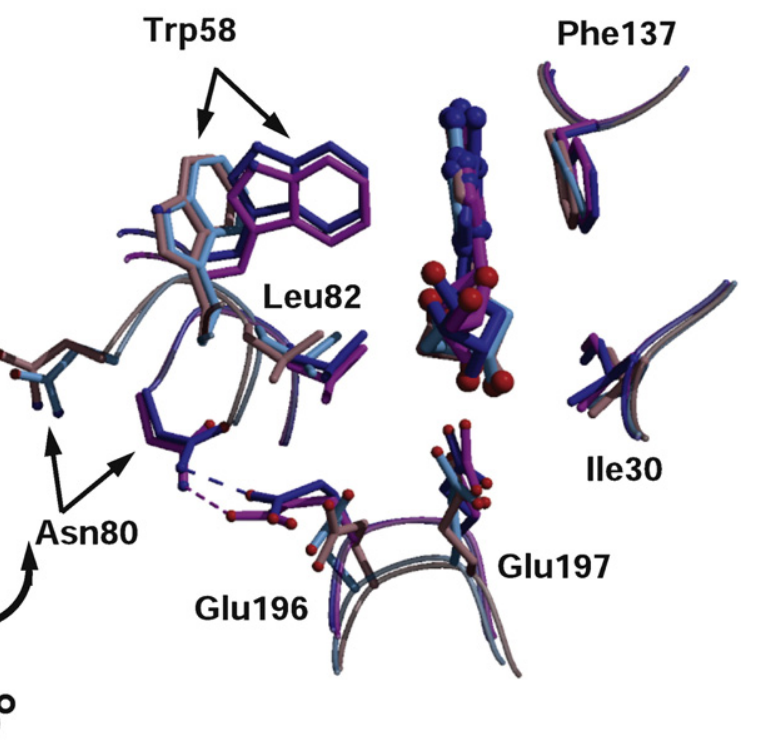

Fig. 7. The overlay of the four complexes highlighting the nucleoside-binding site. (a) Depending on the nature of the donor, dCK adopts either a closed (with ADP) or an open (with UDP) conformation. This affects binding of the nucleoside. In all cases, the adenine base occupies the same area, albeit with $180^{\circ}$ opposite conformation. Tyr86 maintains an interaction with the $3^{\prime}-\mathrm{OH}$ group of the nucleosides, but only in the closed state can a water molecule (green arrow) bridge this residue and the base moiety of the nucleoside. (b) Trp58 changes its conformation dramatically between the open and closed states. 
Unexpected was the observation of two different D-dA conformations: syn with UDP, and anti with ADP. Solution studies reveal that purines undergo rapid inter-conversion between their syn and anti conformations. ${ }^{13}$ One could have surmised that the requirement for optimal binding to the enzyme would select only one of the two conformations. Surprisingly, human dCK can bind both the syn and anti conformations. This is, to our knowledge, the first report of an enzyme that can bind to both conformers (syn and anti) of a nucleoside. This is made possible by the fact that in both binding modes very similar nucleoside-enzyme interactions occur.

Our work reveals that dCK displays substrate promiscuity at both the nucleoside acceptor and nucleotide donor sites. Communication between the two binding sites results in differential states according to the nature of substrates. Large variability in binding mode is possible at the nucleoside acceptor site. While the base moiety always occupies the same space, two binding modes related by a $180^{\circ}$ rotation are possible. The sugar of the nucleoside is much less restricted in its conformation. The conformations observed with UDP represent an inactive state based on a large $5^{\prime}-\mathrm{OH}$ - Glu53 distance. Our work reveals the conformational flexibility of this physiologically important nucleoside kinase and shows how purine substrates of either chirality are accepted as substrates.

\section{Material and Methods}

\section{Site-directed mutagenesis, protein expression, purification, and crystallization}

The $\mathrm{C}_{4}$ S-dCK mutant (C9S/C45S/C59S/C146S) was engineered, over-expressed and purified as described. ${ }^{4,5}$ Briefly, we made the mutant using the QuickChange SiteDirected Mutagenesis Kit from Stratagene using as template the WT-dCK gene cloned into the $\mathrm{pET} 14 \mathrm{~b}$ vector. The BL21(DE3) Escherichia coli strain carrying the recombinant plasmid coding for His-tagged $\mathrm{C}_{4} \mathrm{~S}$-dCK was grown in 2YT medium at $37{ }^{\circ} \mathrm{C}$, induced with $0.1 \mathrm{mM}$ IPTG and harvested after $4 \mathrm{~h}$. After sonication of the cell pellet, the lysate was loaded onto a HisTrap HP column packed with $5 \mathrm{ml}$ of Ni Sepharose high-performance resin (Amersham Biosciences). After loading, the column was washed with $500 \mathrm{ml}$ of buffer containing $20 \mathrm{mM}$ imidazole (pH 7.5), and protein was eluted with $200 \mathrm{mM}$ imidazole (in $50 \mathrm{mM}$ Hepes ( $\mathrm{pH}$ 7.5) and $500 \mathrm{mM} \mathrm{NaCl}$ ). EDTA ( $2 \mathrm{mM}$ ) was added immediately to the eluted protein to avoid degradation by traces of proteases. The His-tag was not cleaved, and the protein was further purified on an S200 gel-filtration chromatography column (Amersham Biosciences) using as gel-filtration buffer $20 \mathrm{mM}$ Hepes (pH 7.5), $200 \mathrm{mM}$ sodium citrate, $2 \mathrm{mM}$ EDTA. The $\mathrm{C}_{4} \mathrm{~S}$ mutant was then crystallized in complex with ADP or UDP and the enantiomeric couple D-dA and L-dA using the vapor-diffusion method in the hanging-drop geometry. D-dA was obtained from Sigma, while L-dA was from ChemGenes Corporation. The complexes were formed by mixing $5 \mathrm{mM}$ nucleoside (D- or L-dA) with $5 \mathrm{mM}$ nucleotide (ADP or UDP) and the enzyme (15-20 mg/ $\mathrm{ml}$ in gel-filtration buffer plus $5 \mathrm{mM} \mathrm{MgCl}_{2}$ ). Crystal- lization trials were also carried out with the nucleotide analogs UppNHp (Jena Biosciences) and AppNHp (Sigma). While crystals were produced, none was of sufficient quality for structural studies.

For the D-dA/ADP complex, the use of the $\mathrm{C}_{4} \mathrm{~S}-\mathrm{dCK}$ mutant proved to be unsuccessful in providing crystals. For a different purpose, we designed a variant of dCK that included the $\mathrm{C}_{4} \mathrm{~S}$ mutation plus the additional mutation of Glu247 to alanine. With this mutant we could produce Xray diffraction quality crystals for the D-dA/ADP complex. All the crystals for the D-/L-dA plus ADP/UDP complexes were obtained from hanging drops $(1 \mu \mathrm{l}$ of protein/nucleoside/nucleotide/ $\mathrm{MgCl}_{2}$ mix plus $1 \mu \mathrm{l}$ of reservoir) using a reservoir solution that contained 0.90 $1.5 \mathrm{M}$ trisodium citrate dihydrate and $100 \mathrm{mM}$ Hepes, $\mathrm{pH}$ 7.5. Formation of X-ray quality diffraction crystals for the four complexes was highly dependent on the temperature at which the drops were incubated. The ADP complexes yielded crystals at room temperature while the UDP complexes crystallized only at $12{ }^{\circ} \mathrm{C}$. Interestingly, by changing the temperature and complex type, dCK produced beautiful crystals characterized by different shapes: the ADP crystals exhibited the typical shape of all our previous crystals for which we determined the structure in space group $C 222_{1}$ or $P 4_{3} 2_{1} 2$, while the UDP crystals were shaped like rice grains.

\section{Crystallographic data collection and processing}

Crystals were transferred to a cryoprotectant solution, consisting of mineral oil (Sigma), mounted in loops, and frozen by immersing them directly in liquid nitrogen. Xray data for the D-dA/ADP crystal were collected at the Advanced Photon Source, Argonne National Laboratories using the SERCAT beamline ID-22, while those for L-dA/ ADP, D-dA/UDP and L-dA/UDP were collected in-house using RAXIS-IV as detector. The data were indexed, scaled and merged using XDS and XSCALE (see Table 1 for data collection statistics). ${ }^{14}$

\section{Structure determination and refinement}

For the two ADP-containing complexes we could commence directly by applying rigid body refinement. For the D-dA/ADP structure our initial model was PDB Code 1P60 while for the L-dA/ADP structure crystallized in space group $P 4_{3} 2_{1} 2$ it was PDB Code $1 P 5 Z$. For the two complexes with UDP it was necessary to perform molecular replacement, done with Molrep ${ }^{15}$ with $1 \mathrm{P} 5 \mathrm{Z}$ as the search model. In order to prepare a good starting model and avoid any possible bias during refinement, residues 55-82 were deleted from each monomer of structure 1P60 (which is a dimer) and from structure 1P5Z (a monomer). This area follows the nucleophilic base Glu53 and includes the insert region. Moreover, for the starting model of the UDP structures, residues 238-254 were also deleted because our previous work revealed that this region is sensitive to the identity of the diphosphate. Restrained refinement for the D-dA/ADP and L-dA/ADP structures was carried out using REFMAC $^{16}$ (for the L-dA/ADP complex, data scaled well to $1.76 \AA$, but refinement was best to $2.2 \AA$ ). Since both UDP structures showed perfect twinning (see Table 1 for twinning fraction of data sets), refinement for these two structures was done using SHELXL by use of conjugate-gradient least-squares refinement. ${ }^{17}$ The electron density map ( $2 F_{\mathrm{obs}}-F_{\text {calc }}$ and omit) for the nucleosides $\mathrm{D}-\mathrm{dA}$ and $\mathrm{L}-\mathrm{dA}$ in the ADP and UDP complexes is shown 
in (Supplementary Data Fig. S4). Arg128, an active site residue, is in the disallowed region of the Ramachandran plot. This is a common feature for this enzyme.

\section{Steady-state kinetic assay}

A spectroscopic enzyme-coupled assay was used to determine the activity of both WT, $\mathrm{C}_{4} \mathrm{~S}$ and $\mathrm{C}_{4} \mathrm{~S}+\mathrm{E} 247 \mathrm{~A}$ $\mathrm{dCK}$ as described ${ }^{18}$ in $50 \mathrm{mM}$ Tris- $\mathrm{HCl}$ (pH 7.5), $100 \mathrm{mM}$ $\mathrm{KCl}$, and $5 \mathrm{mM} \mathrm{MgCl}$ at $37^{\circ} \mathrm{C}$ with $0.35 \mu \mathrm{M} \mathrm{dCK}, 1 \mathrm{mM}$ ATP-Mg and $1 \mathrm{mM}$ UTP-Mg. D-dA and L-dA concentrations were varied between $50 \mu \mathrm{M}$ and $600 \mu \mathrm{M}$ when ATP was used as phosphate donor and $5 \mu \mathrm{M}$ to $100 \mu \mathrm{M}$ with UTP (Table 2).

\section{Fluorescence}

Intrinsic tryptophan fluorescence measurements of proteins were recorded using a Quanta Master spectrofluorimeter (Photon Technology International, London, Ontario, Canada). The excitation wavelength was $295 \mathrm{~nm}$, to ensure excitation of only the tryptophan, with slits of $1 \mathrm{~nm}$ for excitation and $4 \mathrm{~nm}$ for emission. Emission was recorded between $310 \mathrm{~nm}$ and $350 \mathrm{~nm}$ in steps of $1 \mathrm{~nm}$ with an integration time of $1 \mathrm{~s}$ at $25^{\circ} \mathrm{C}$.

All solutions were prepared in $0.05 \mathrm{M}$ sodium phosphate buffer, $\mathrm{pH}$ 7.5. The temperature of the samples was maintained at $25{ }^{\circ} \mathrm{C}$ using a thermostatically controlled cell holder. Fluorescence spectra were taken in a two-step process, first the spectrum of the protein $(30 \mu \mathrm{M})$ was taken, and then UTP or ATP was added, and an additional spectrum taken. No significant change in signal was observed upon addition of $\mathrm{dA}$, either to the apo or the UDP-complexed dCK.

\section{Protein Data Bank accession codes}

Structures have been deposited in the PDB with accession codes 2ZI3 for the D-dA/ADP, 2ZI4 for the L$\mathrm{dA} / \mathrm{ADP}, 2 \mathrm{ZI} 6$ for the D-dA/UDP, and 2ZI5 for the L-dA/ UDP complex.

\section{Acknowledgements}

We thank Dr Andrey Lebedev (University of York, UK), Dr Bernard Santarsiero (University of Illinois at Chicago) and Professor George Sheldrick (University of Göttingen, Germany) for helpful discussions regarding the twinned data sets, and the staff at the SER-CAT beamline for help in data collection. We acknowledge Dr Christophe Mathé for helpful discussion on nucleoside conformations in solution. This work was supported by an NIH grant (to E.S., S.H., and A.L.) and the Max-PlanckSociety (to M.K.).

\section{Supplementary Data}

Supplementary data associated with this article can be found, in the online version, at doi:10.1016/ j.jmb.2008.02.061

\section{References}

1. Shewach, D. S., Reynolds, K. K. \& Hertel, L. (1992). Nucleotide specificity of human deoxycytidine kinase. Mol. Pharmacol. 42, 518-524.

2. Shewach, D. S., Liotta, D. C. \& Schinazi, R. F. (1993). Affinity of the antiviral enantiomers of oxathiolane cytosine nucleosides for human 2'-deoxycytidine kinase. Biochem. Pharmacol. 45, 1540-1543.

3. Sabini, E., Ort, S., Monnerjahn, C., Konrad, M. \& Lavie, A. (2003). Structure of human dCK suggests strategies to improve anticancer and antiviral therapy. Nature Struct. Biol. 10, 513-519.

4. Sabini, E., Hazra, S., Konrad, M., Burley, S. K. \& Lavie, A. (2007). Structural basis for activation of the therapeutic L-nucleoside analogs 3TC and troxacitabine by human deoxycytidine kinase. Nucleic Acids Res. 35, 186-192.

5. Sabini, E., Hazra, S., Konrad, M. \& Lavie, A. (2007). Nonenantioselectivity property of human deoxycytidine kinase explained by structures of the enzyme in complex with L- and D-nucleosides. J. Med. Chem. 50, 3004-3014.

6. Zhang, Y., Secrist, J. A., III \& Ealick, S. E. (2006). The structure of human deoxycytidine kinase in complex with clofarabine reveals key interactions for prodrug activation. Acta Crystallogr. D, 62, 133-139.

7. Godsey, M. H., Ort, S., Sabini, E., Konrad, M. \& Lavie, A. (2006). Structural basis for the preference of UTP over ATP in human deoxycytidine kinase: illuminating the role of main-chain reorganization. Biochemistry, 45, 452-461.

8. Krawiec, K., Kierdaszuk, B., Eriksson, S., MunchPetersen, B. \& Shugar, D. (1995). Nucleoside triphosphate donors for nucleoside kinases: donor properties of UTP with human deoxycytidine kinase. Biochem. Biophys. Res. Commun. 216, 42-48.

9. Hughes, T. L., Hahn, T. M., Reynolds, K. K. \& Shewach, D. S. (1997). Kinetic analysis of human deoxycytidine kinase with the true phosphate donor uridine triphosphate. Biochemistry, 36, 7540-7547.

10. Turk, B., Awad, R., Usova, E. V., Bjork, I. \& Eriksson, S. (1999). A pre-steady-state kinetic analysis of substrate binding to human recombinant deoxycytidine kinase: a model for nucleoside kinase action. Biochemistry, 38, 8555-8561.

11. Mani, R. S., Usova, E. V., Cass, C. E. \& Eriksson, S. (2006). Fluorescence energy transfer studies of human deoxycytidine kinase: role of cysteine 185 in the conformational changes that occur upon substrate binding. Biochemistry, 45, 3534-3541.

12. Hughes, T. L., Hahn, T. M., Reynolds, K. K. \& Shewach, D. S. (1997). Kinetic analysis of human deoxycytidine kinase with the true phosphate donor uridine triphosphate. Biochemistry, 36, 7540-7547.

13. Saenger, W. (1984). In Principles of Nucleic Acid Structure (Charles, R. \& Cantor, E., eds), SpringerVerlag, New York.

14. Kabsch, W. (1993). Automatic processing of rotation diffraction data from crystals of initially unknown symmetry and cell constants. J. Appl. Cryst. 26, 795-800.

15. Vagin, A. \& Teplyakov, A. (1997). MOLREP: an automated program for molecular replacement. J. Appl. Crystallogr. 30, 1022-1025.

16. Murshudov, G. N., Vagin, A. A. \& Dodson, E. J. (1997). Refinement of macromolecular structures by the maximum likelihood method. Acta Crystallogr. D, 53, 240-255.

17. Sheldrick, G. \& Schneider, T. (1997). SHELXL: highresolution refinement. Methods Enzymol. 277, 319-343. 
18. Agarwal, K. C., Miech, R. P. \& Parks, R. E. (1978). Guanylate kinases from human erythrocytes, hog brain, and rat liver. Methods Enzymol. 51, 483-490.

19. Kraulis, P. J. (1991). MOLSCRIPT: a program to produce both detailed and schematic plots of protein structures. J. Appl. Crystallogr. 24, 946-950.
20. Esnouf, R. (1997). An extensively modified version of molscript that includes greatly enhanced coloring capabilities. J. Mol. Graph. 15, 133-138.

21. Merrit, E. A. \& Murphy, M. E. P. (1994). Raster3D Version 2.0 - a program for photorealistic molecular graphics. Acta Crystallogr. D, 50, 869-873. 